\title{
An Intersystem Transition in the First Spectrum of Beryllium
}

\author{
W. R. Bozman, C. H. Corliss, W. F. Meggers, and R. E. Trees
}

\begin{abstract}
The intersystem line from the transition $2 s^{2}{ }^{1} \mathrm{~S}_{0}-2 s 2 p{ }^{3} \mathrm{P}_{1}$ has been observed in the first spectrum of beryllium. Wavelength measurements give $4548.538 \pm .002 \mathrm{~A}$, which agrees with the predicted value of $4548.29 \pm .4 \mathrm{~A}$. The energy of the ${ }^{3} \mathrm{P}_{1}^{\circ}$ level is thus determined to be $21,978.92+.01 \mathrm{~K}$ above the ground state, and the value $x$ to be added to all of the triplet terms of Be I tabulated in "Atomic Energy Levels" is $-1.18 \mathrm{~K}$. Intensity measurements show that the intensity ratio of the singlet resonance line Be I 2348.61 A, to the intersystem line is about $3 \times 10^{7}$.
\end{abstract}

\section{Introduction}

In atomic or ionic spectra displaying two or more systems of terms differing in multiplicity, intersystem transitions are forbidden by the selection rules for pure $L S$ coupling. However, the coupling is never strictly pure, and intersystem transitions of considerable intensity have been observed in many spectra, thus fixing the values of all derived spectral terms relative to the zero-energy ground state. If intersystem transitions are not observed, the values of a term system, not including the ground state, will remain uncertain relative to that state by a constant amount, $x$. Examples of such uncertainties are found in the first two volumes of Atomic Energy Levels [1]. ${ }^{1}$

Intersystem transitions produce fairly strong lines in spectra of the heavy elements but become progressively weaker in the spectra of light elements. The intersystem combination ${ }^{1} \mathrm{~S}_{0}-{ }^{3} \mathrm{P}_{1}$ in $\mathrm{He} \mathrm{I}$ was first detected at 591 A in 1924 by Lyman [2] and later confirmed by Hopfield [3], by Paschen [4], and by Suga [5]. The intensity of this line according to Hopfield [3] and Suga [5] is comparable with that of ${ }^{1} \mathrm{~S}_{0}-8{ }^{1} \mathrm{P}_{1}$ or ${ }^{1} \mathrm{~S}_{0}-9{ }^{1} \mathrm{P}_{1}$, although Suga [5] says that in a continuous discharge in a water-cooled tube containing helium at $7 \mathrm{~mm}(\mathrm{Hg})$ pressure it is comparable with the third or fourth member of this singlet series. In any case, the intensity of the intersystem transition is only a small fraction of that characteristic of $584 \mathrm{~A}$, the first line of this series.

The transitions, ${ }^{1} \mathrm{~S}_{0}-{ }^{3} \mathrm{P}_{1}$, in analogous Li II, Be I, B II, C III, N Iv spectra have not been observed. In the case of $\mathrm{Be} I$, the wavelength of this resonance line was first calculated from series limits by Paschen and Kruger [6] to be $4547.88 \pm 0.4 \mathrm{~A}$, and later when Paschen [7] revised the limit of the singlet series he predicted the wavelength should be $4548.29 \mathrm{~A}$. In 1929 Paton and Nusbaum [8] reported a line at 4553.07 A to which they assigned this classification, but their result is far beyond the uncertainty quoted above and has not been confirmed. The intersystem resonance line $2 s^{2}{ }^{1} \mathrm{~S}_{0}-2 s 2 p{ }^{3} \mathrm{P}_{1}$ of $\mathrm{Be} \mathrm{I}$ has been observed by the present authors, who here report the measured wavelength and estimated relative intensity.

${ }_{1}^{1}$ Figures in brackets refer to literature references at the end of this paper.

\section{Experimental Procedure}

The observation of extremely weak lines, such as the intersystem resonance line of Be I, requires an intense light source free from molecular bands and other spectral background and an efficient spectrograph of high linear dispersion. The light source adopted in this case was a 5-amp d-c arc between pure beryllium electrodes. To eliminate $\mathrm{Be} \mathrm{O}$ bands that lie in the violet region under observation the source was operated in an enclosure [9] filled with helium. The arc was focused on the slit of a stigmatic concave-grating spectrograph [10]. An excellent 30,000-line/in. grating ruled by R. W. Wood produces a first-order spectrum with a reciprocal dispersion of $2.18 \mathrm{~A} / \mathrm{mm}$ at $4550 \mathrm{~A}$. A 25-micron slit was used, and a glass filter was placed in front of the slit to absorb the overlapping second-order spectrum. The Be I intersystem line was photographed on Eastman 103-O plates with exposures of 20 to $100 \mathrm{~min}$. When the are is operated in air, this line is lost in the bands of $\mathrm{Be} \mathrm{O}$.

To make sure that the observed line was not a false line produced by the grating, a second series of exposures was made with a large quartz-prism spectrograph having a reciprocal dispersion of 6.3 $\mathrm{A} / \mathrm{mm}$ at $4550 \mathrm{~A}$. The existence of the line was confirmed in these spectra.

\section{Experimental Results}

The spectrum line produced by the intersystem combination is shown in figure 1 just to the left of the fifth left-side ghost of Be I, 4572.67 A. The average of four measurements on two grating spectrograms is $4548.538 \pm .002 \mathrm{~A}$. This is within the uncertainty of the predicted value $4548.29 \pm .4 \mathrm{~A}$ [7]. The energy of the $2 s 2 p^{3} \mathrm{P}_{1}$ level is thus determined to be $21,978.92 \pm .01 \mathrm{~K}^{2}$ above the ground state and the value $x$ to be added to all of the triplet terms of Be I tabulated in Atomic Energy Levels [1] is $-1.18 \mathrm{~K}$.

An attempt was made to determine the intensity of the intersystem line relative to the more prominent lines of Be I. On the assumption of photographic reciprocity of time and intensity, a series of

${ }^{2}$ In accordance with the recommendation of the Joint Commission for Spec troscopy, the name "kayser" is used for the unit of wave number, $1 \mathrm{~K}=1 \mathrm{~cm}^{-1}$. 


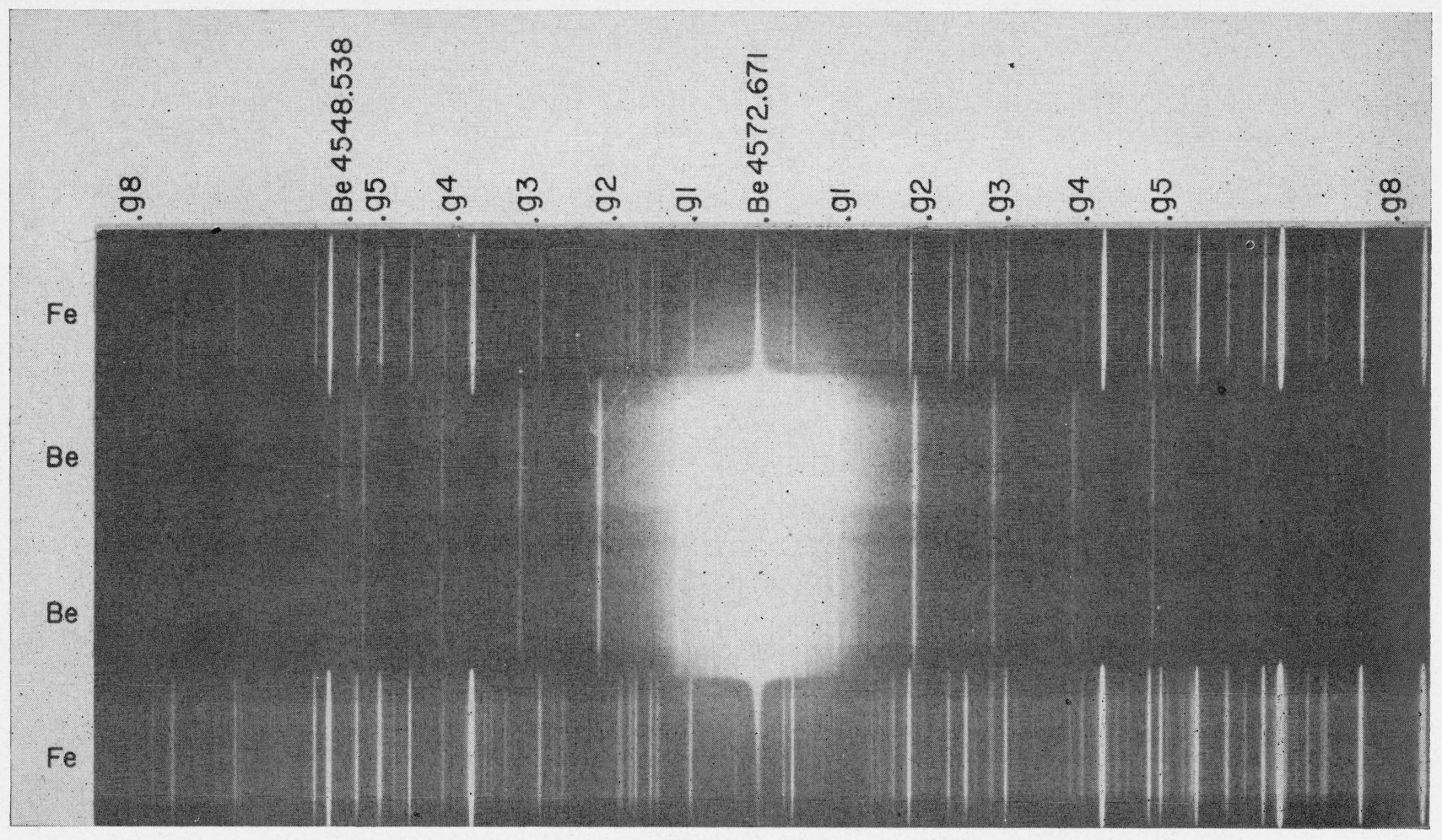

Figure 1. Intersystem resonanceline Be I, 4548.538 A.

timed exposures indicated that the ratio of the intensity of the parent line to the fifth ghost from this grating was about 100,000 . From a comparison of the intersystem line with the ghosts of Be I, $4572.67 \mathrm{~A}$, the intensity ratio $\frac{\mathrm{I}_{4572.67 \mathrm{~A}}}{\mathrm{I}_{4548.54 \mathrm{~A}}}$ is about 400,000 . Data taken from standardized arc spectrograms of $\mathrm{Be}$ diluted in $\mathrm{Cu}$ to eliminate self-absorption show that the intensity ratio of the $\left(2 s^{2}{ }^{1} \mathrm{~S}_{0}-\right.$ $\left.2 s 2 p^{1} \mathrm{P}_{1}^{\circ}\right)$ resonance line, Be $\mathrm{I}, 2348.61 \mathrm{~A}$, to $\left(2 s 2{ }^{1} \mathrm{P}_{1}^{\circ}-2 s 3 d^{1} \mathrm{D}_{2}\right)$ Be I, $4572.67 \mathrm{~A}$ is about 70 . Therefore, the experimentally determined ratio of the intensity of the singlet resonance line to that of the intersystem is about $3 \times 10^{7}$.

Assuming that the number of atoms in any excited level is determined by the Boltzmann distribution, the theoretical ratio is $[11,12]$

$$
\begin{aligned}
& r=\frac{9}{2}\left[\frac{\left({ }^{1} \mathrm{P}_{1}\right)-\left({ }^{1} \mathrm{~S}_{0}\right)}{\left({ }^{3} \mathrm{P}_{1}\right)-\left({ }^{1} \mathrm{~S}_{0}\right)}\right]^{4}\left[\frac{\left({ }^{1} \mathrm{P}_{1}\right)-\left({ }^{3} \mathrm{P}_{1}\right)}{\left({ }^{3} \mathrm{P}_{2}\right)-\left({ }^{3} \mathrm{P}_{0}\right)}\right]^{2} \\
& \exp \left[-\frac{1.439\left[\left({ }^{1} \mathrm{P}_{1}\right)-\left({ }^{3} \mathrm{P}_{1}\right)\right]}{T}\right],
\end{aligned}
$$

where the levels in parentheses indicate the corresponding energy in kaysers. The temperature, $T$, is in degrees Kelvin. For an arc temperature of $5,000^{\circ} \mathrm{K}$, the calculated value of the intensity ratio is $8 \times 10^{6}$. By use of the parameter $\lambda$ introduced by King and Van Vleck [11] (assuming the value $\lambda=0.8$, which is an average value in the heavy elements), the calculated ratio is increased to $1.2 \times 10^{7}$. For light elements such as Be, one must also consider the effects of spin-spin and spin-other-orbit interactions on the intensity ratio, which can be done approximately by replacing the difference $\left({ }^{3} \mathrm{P}_{2}\right)-\left({ }^{3} \mathrm{P}_{0}\right)$ by $2\left({ }^{3} \mathrm{P}_{2}\right)+\left({ }^{3} \mathrm{P}_{0}\right)-3\left({ }^{3} \mathrm{P}_{1}\right)$. This correction reduces the calculated value to $7 \times 10^{6}$. The calculation is most sensitive to the value assumed for the temperature; a 10-percent change in $T$ will alter $r$ by a factor on the order of 2 .

The difference between the calculated value $\left(7 \times 10^{6}\right)$ and the experimental value $\left(3 \times 10^{7}\right)$ may be outside the limits of experimental error, but better agreement could hardly be expected in view of the untested assumptions.

\section{References}

[1] C. E. Moore, Atomic energy levels, NBS Circ. 467, I (1949) II (1952).

[2] T. Lyman, Astrophys. J. 60, 1 (1924)

[3] J. J. Hopfield, Astrophys. J. 7\%, 133 (1930)

[4] F. Paschen, Sitz. ber. deu. Akad. Wiss. Berlin, 30, 662 (1929)

[5] T. Suga, Sci. Pap. Inst. Phys. Chem. Res. (Tokyo) 34, $16 \quad 1937$ )

[6] F. Paschen and P. G. Kruger, Ann. Physik [5] 8, 1005 (1931).

[7] F. Paschen, Ann. Physik [5] 12, 509 (1932).

[8] R. F. Paton and R. E. Nusbaum, Phys. Rev. 33, 1093 (A) (1929).

[9] H. D. Curtis, J. Opt. Soc. Am. and Rev. Sci. Instr. 8, 697 (1924)

[10] W. F. Meggers and K. Burns, BS Sci. Pap. 18, 191 (1922) S441.

[11] G. W. King and J. H. Van Vleck, Phys. Rev. 56, 464 (1939).

[12] P. J. Rubenstein, Phys. Rev. 58, 1007 (1940).

Washington, January 28, 1953. 